\title{
Profile of Medically Compromised Children Attending the Pediatric Dentistry Department of the Casablanca Dental Consultation and Treatment Center
}

\author{
Bensouda Sana, Elasmar Mouna ${ }^{*}$, Aljalil Zineb, El Moutaki Soukaina, Benjelloun Sarah, \\ Hamza Mouna, Elarabi Samira
}

Department of Pediatric Dentistry, Faculty of Dental Medicine, University Hassan II, Casablanca, Morocco

Email address:

bensoudas2001@yahoo.fr (B. Sana), mouna.elasmar2000@gmail.com (E. Mouna)

${ }^{*}$ Corresponding author

To cite this article:

Bensouda Sana, Elasmar Mouna, Aljalil Zineb, El Moutaki Soukaina, Benjelloun Sarah, Hamza Mouna, Elarabi Samira. Profile of Medically Compromised Children Attending the Pediatric Dentistry Department of the Casablanca Dental Consultation and Treatment Center. International Journal of Dental Medicine. Vol. 5, No. 1, 2019, pp. 35-39. doi: 10.11648/j.ijdm.20190501.16

Received: March 11, 2019; Accepted: April 26, 2019; Published: June 11, 2019

\begin{abstract}
Advances in pediatric medicine and medical technology have led to a spectacular reduction in morbidity and mortality among medically compromised children. As a result, dentists are facing the challenge of treating and managing more children with systemic conditions meeting affecting the dental treatment. Objective: To evaluate the medical profile of medically compromised children followed in the Pediatric Dentistry Department of the Casablanca Dental Consultation and Treatment Center and to assess their oral health. Materials and methods: This is a cross-sectional study conducted with 529 children treated in the pediatric dentistry department between 16 November 2016 and 16 January 2017. The general condition status, oral hygiene and dental condition were collected based on the anamnesis and oral examination. Results: From the 529 children included in the study, $1 / 3$ of them have one or more systemic conditions. Patients with special needs represent $27.2 \%$ of the total of medically compromised children. The DMFT and dmft index ware respectively of 6.42 and 3.44 . The average plaque index was 1.04. Conclusion: The oral status of medically compromised children is alarming with a large number of decayed teeth and few treated teeth. This is related to the quality of oral hygiene measures introduced by children and their parents, as well as the impact of the medical condition.
\end{abstract}

Keywords: Medically Compromised Children, Dental Consultation, Oral Health

\section{Introduction}

A poor oral health can have a considerable impact on the general health status and quality of life of otherwise healthy children, their effect on those with chronic and acute illnesses can be much more serious. In some cases it might lead to increased risks of life-threatening complications [1]. In addition, dental pain and infections can further compromise the quality of life of children with mental, developmental or physical disabilities [1].

Medically compromised children have a higher risk to develop oral disease, because the disease itself may include oral manifestations [2, 3]. Furthermore, the treatment or medication prescribed may lead to a decreased in host resistance and side effects in the oral cavity. Untreated dental disease in medically compromised children can significantly affect their general health and quality of life. Indeed, in some conditions an acute dental infection can be fatal [4-6].

The evaluation of the medical profile of children consulting at university hospitals is of great importance at the global level. In fact, several studies have been conducted in this direction, most of which are focusing on the medical profile of adult patients [4, 5, 7-10]. However, few studies have addressed the subject of the medical profile of children consulting for dental care $[1,11,12]$. The situation is similar in Morocco. Very few studies have examined the medical profile of children consulting for dental care.

The main objective of this study is to evaluate the medical profile of children consulting and following up in the pediatric dentistry department of the Casablanca Dental Consultation 
and Treatment Center, and to assess their oral health.

\section{Participants and Methods}

This is a cross-sectional study conducted in the pediatric dentistry department of the Dental Consultation and Treatment Center in Casablanca. The study covered 529patients managed at the pediatric dentistry department from 15 November 2016 to 16 January 2017.

The Institutional ethical committee approved this research and parental consent of all patients was obtained before conducting the study.

Data collection was carried out using a questionnaire consisting of four successive parts: the patient identification, the patient's medical profile, the children's dietary habits and oral hygiene practices and the patient oral health status.

Patient identification included information on age, sex and socioeconomic status.

Child's general condition was determined by the presence or absence of a general pathology. Medically compromised conditions were classified into12 categories: hematological and oncological diseases, cardiovascular diseases, endocrine disorders, infectious diseases, nervous system disorders, respiratory diseases, gastric diseases, dermatological diseases, multi-systemic diseases, autoimmune diseases, patients with special needs and others.

According to the compromised medical condition, the modified ASA classification for dentistry was used to determine risk during dental treatment [13].

Other information on current medication and chief complaint were obtained.

Dietary habits and oral hygiene practices were also assessed in the questionnaire; type of diet, sweetened beverages/snacks and their frequency, number of meals and dental brushing.

Then, all subjects were examined for dental caries, and oral hygiene status. Caries status was evaluated using the decayed, extracted and filled primary teeth (deft) index and decayed, missing, or filled (DMFT) permanent teeth (1997) [14]. The SILNESS and LOE plaque index(1964) used to ascertain oral hygiene status, which is recorded as excellent, good, fair or poor $[15,16]$.

The results were analyzed at the Laboratory of Epidemiology and Bio-statistics of the Faculty of Dentistry of Casablanca.

\section{Results}

Of the 529 pediatric patients included in this study, 240
(45.4\%) were female and $289(54.6 \%)$ were male. The patients had an age range of 2-16 years with a mean age of $8.46 \pm 3.04$ years.

In this sample, 180 patients (34\%) were afflicted with one or more medically compromised conditions and 349 (66\%) were categorized as healthy (no medical conditions reported). Prevalence of medication use in medically compromised children was $66.7 \%$.

In the present study, the most frequently encountered medical condition category was patients with special needs followed in descending order by, multisystemic diseases, hematological and oncological diseases, cardiovascular diseases, respiratory diseases, others, nervous system disorders, dermatological diseases, endocrine disorders, autoimmune diseases, infectious diseases and gastric diseases, respectively (Table 1).

Table 2 gives the distribution of patients with specific needs by type of disability.

The percentage of patients medically compromised in each ASA category was: ASA II 46.7\% (84), ASA III 20.6\% (37), and ASA IV 32.8\% (59).

The chief complaint has been classified into two types: either the patient is referred by his attending physician, or he consults for his own interest for either an emergency or an oral rehabilitation (Table 3).

Regarding previous dental visits, 94 (52.2\%) of medically compromised children have never consulted a dentist, and 86 (47.8\%) of children have already visited a dentist, but (56.98\%) of them have been referred directly to the Pediatric Dentistry Department without any prior treatment.

Data of dietary habits of medically compromised children showed that the number of meals was more than four meals per day for $111(61.7 \%)$ children, and $55 \%$ of children consume sweetened drinks, outside meals (Table 4).

Concerning oral hygiene practices, approximately $76,7 \%$ (138) of subjects reported regularly brushing their teeth less than 2 times/day for $63.8 \%$ and greater than 2 times/day for $36.2 \%$. No dental brushing was reported in $23.3 \%$ (42) of children (Table 5).

The mean DMFT index of medically compromised children was $6.42 \pm 5.29$ with a number of decayed teeth (1.78), and the mean dmft index was $3.44 \pm 4.09$ with a number of decayed teeth (3.92).

The mean plaque index value of the examined children was $1.04 \pm 0.43$ and the oral hygiene was qualified as good in $37.1 \%$, fair in $58.4 \%$ and poor in $4.5 \%$.

Table 1. Disease categories and frequency of diseases.

\begin{tabular}{|c|c|c|c|}
\hline Disease category & $\mathbf{N}$ & $\%$ & Type of diseases \\
\hline $\begin{array}{l}\text { Hematological and } \\
\text { oncological diseases }\end{array}$ & 35 & $19,4 \%$ & $\begin{array}{l}\text { Thalassemia, Sickle cell disease, Hemophilia A and B, Purpura, Iron deficiency anemia and } \\
\text { Hypochrome, Thrombocytopenia, Pancytopenia, Leukemia, lymphomas, Neuroblastoma, } \\
\text { Nephroblastoma, Medulloblastoma... }\end{array}$ \\
\hline Cardiovascular diseases & 13 & $7,2 \%$ & Congenital heart disease, Valve stenosis, Acute rheumatic fever, Bicuspidy of the aortic valve... \\
\hline Endocrine disorders & 5 & $2.8 \%$ & Insulin-dependent diabetes, Non-insulin-dependent diabetes, Growth retardation.... \\
\hline Infectious diseases & 2 & $1.1 \%$ & Tuberculosis-meningeal, Sinusitis...... \\
\hline Nervous system disorders & 8 & $4.4 \%$ & Epilepsy, Hydrocephaly...... \\
\hline Gastric diseases & 0 & $0 \%$ & Gastritis...... \\
\hline
\end{tabular}




\begin{tabular}{llll}
\hline Disease category & N & $\%$ & Type of diseases \\
\hline Dermatological diseases & 7 & $3.9 \%$ & $\begin{array}{l}\text { Congenital ichthyosis, } \\
\text { Epidermolysis bullosa, Ectodermal dysplasia, Cutaneous allergy.... }\end{array}$ \\
Multisystemic diseases & 37 & $20.6 \%$ & $\begin{array}{l}\text { Patient with more than one systemic pathology } \\
\text { Autoimmune diseases }\end{array}$ \\
Patients with specific needs & 4 & $2.2 \%$ & Immune deficiency, juvenile arthritis, rheumatoid arthritis..... \\
Others & 10 & $5.56 \%$ & Renal diseases, Allergy to dust, Behavioral disorders, G6PD deficiency..... \\
\hline
\end{tabular}

Table 2. Distribution of patients with specific needs by type of disability.

\begin{tabular}{lll}
\hline Patients with specific needs & No & \% \\
\hline Autism & 12 & $24,49 \%$ \\
Cerebral palsy & 16 & $32.65 \%$ \\
Mental retardation & 11 & $22.45 \%$ \\
Psychomotor deficiency & 5 & $10.20 \%$ \\
Deaf and dumb & 1 & $2.04 \%$ \\
Trisomy 21 & 4 & $8.16 \%$ \\
Total & 49 & $100 \%$ \\
\hline
\end{tabular}

Table 3. Chief Complaint of Medically Compromised Patients.

\begin{tabular}{lll}
\hline & No & \% \\
\hline Addressed by the treating physician & 107 & $59.4 \%$ \\
Dental emergencies & 36 & $20.0 \%$ \\
Rehabilitation of the oral cavity & 37 & $20.6 \%$ \\
Total & 180 & $100 \%$ \\
\hline
\end{tabular}

Table 4. Dietary Habits of Medically Compromised Patients.

\begin{tabular}{lll}
\hline & No & \% \\
\hline Meals per day & & \\
$\leq 4$ times / day & 69 & $38.3 \%$ \\
$>4$ times / day & 111 & $61.7 \%$ \\
Sweet drinks & & \\
Yes & 99 & $55.0 \%$ \\
No & 81 & $45.0 \%$ \\
\hline
\end{tabular}

Table 5. Oral Hygiene Practices of Medically Compromised Patients.

\begin{tabular}{lll}
\hline & No & \% \\
\hline Dental brushing & & \\
Yes & 138 & $76.7 \%$ \\
No & 42 & $23.3 \%$ \\
Brushing frequency & & \\
$<2$ times/day & 88 & $63.8 \%$ \\
$\geq 2$ times/day & 55 & $36.2 \%$ \\
\hline
\end{tabular}

\section{Discussion}

In dentistry, the patient "at risk" is patient that present one or more diagnosed or suspected medical conditions, treated or not. The interactions between oral health and general health are multiple and bidirectional. Dental management of the medically compromised patient requires acquisition of a complete health history of the patient [4]. Because, some medical situations require precautions or even therapeutic abstention, and even if dental treatment has been perfectly carried out, recurrences and/or complications may occur due to the patient's general state $[4,5]$.

The prevalence of the medically compromised children in this study was $34 \%$. This value is significantly lower than those reported in other studies. It was $66 \%$ in Boston [12] and $54.66 \%$ in Saudi Arabia [1]. This difference can be explained by geo-demographic, ethnic and socio-economic differences [10].

In the present study, the most frequently encountered disease category was that of patients with special needs $(27.2 \%)$ with a predominance of children with cerebral palsy $(32.65 \%)$, followed by the category of multi-systemic diseases $(20.6 \%)$. The prevalence of category of hematological and oncological diseases was (19.4\%), which could be explained by the close collaboration between the pediatric dentistry department and the hematology and pediatric oncology department. The other categories of cardiovascular diseases $(7.2 \%)$, respiratory diseases $(5.6 \%)$, nervous system diseases (4.4\%), and dermatological diseases $(3.9 \%)$ had a low prevalence.

The increasing prevalence of chronic diseases in this study has also led to a growing demand for medications, with $66.7 \%$ of medically compromised children taking one or more drugs. The concomitant use of a variety of drugs by patients requires a perfect knowledge of drugs by dentists to avoid drug interactions during prescriptions and to anticipate the undesirable effects of some drugs [4].

The ASA 2 score was obtained for $46.7 \%$ of children, ASA 3 for $20.6 \%$, and ASA 4 for $32.8 \%$. This classification allows the dentist to identify patients who can be treated safely during dental care, as well as those whose treatment involves a higher risk $[7,6,13]$.

In the present study, $59.4 \%$ of medically compromised children are referred by their attending physicians for comprehensive care, $20.6 \%$ of these children for oral cavity rehabilitation, and $20 \%$ who consult for an emergency. No patient has consulted just for a routine examination or systematic visit, regardless of their socio-economic level. The study carried out in the University of Hacettepe [11], reports that the systematic visit or routine examination was the first chief complaint of their patients $(46.4 \%)$.

This may be explained by the fact that dental care for medically compromised children is often neglected. The preoccupation of parents about the principal medical condition often leads to neglect of other aspects of the child's overall health $[11,17]$.

This situation remains alarming.

Serious work must be done with attending physicians as part of collaboration between dentists and physicians to encourage regular oral check up, and facilitate referral of at risk children to pediatric dental services $[2,11]$. Children with chronic childhood diseases are more prone to developing oral diseases [3], they should have a dental examination every 3 to 6 months, even if they have no dental problems. This systematic visit would raise parents' awareness of the importance of oral hygiene and the impact of poor oral health on systemic diseases. Furthermore, those children 
should receive the highest priority for comprehensive preventive dental care from an early age $[2,11]$.

In this study, $61.7 \%$ of medically compromised children consume more than four meals a day. This high meal consumption could be explained by the attitude of parents who try to compensate their children's illness with treats and also to solve the problem of staturo-weight slowdown that affects many of these patients.

Sugars are most important cause of dental caries. Frequent consumption of carbohydrate containing snacks between meals is known to increase the amount of dental caries [18]. Despite the knowledge of the relationship between sugar abuse and the appearance of dental decay, the average consumption of sweetened drinks remains relatively high in our sample 55\%. The UFSBD "French Union for Oral Health" recommends that children should not exceed four meals a day without any intake of sweetened drinks or soda even "light" in order to maintain a good oral health [18].

The role of tooth brushing in prevention of dental diseases has been well established [11, 19]. Unfortunately, $50 \%$ of the children examined reported that they did not brush their teeth, while the others had the habit of brushing their teeth irregularly. These results can be explained by the lack of awareness among these children and lack of knowledge from parents about the risks of poor oral hygiene on their overall health.

In this study, $52.2 \%$ of children with medical condition have never seen a dentist, and $56.98 \%$ of children who have already consulted a dentist are not cared for. Studies have shown that many general dental practitioners were quite reluctant to treat patients with chronic diseases [3, 14].

This study reported a DMFT index of 6.42 (with a component " $D$ " = 1.78) and a dmft index $=3.44$ (with a component " $d "=3.92$ ). This value is higher than that found in the study conducted in Haceteppe [11]. This underlines, once again, that the oral state of the Moroccan medically compromised child is alarming with a large number of decayed teeth and few treated teeth.

\section{Conclusion}

Dentists are increasingly treating more medically complex patients who require complex services with more challenging dental treatment planning.

Knowing about systemic diseases and medications will help dental practitioners to be aware of life-threatening situations that may occur during dental treatment.

\section{Recommendations}

The general pediatrician plays an important role in educating medically compromised children and their families about the importance of good dental health, encouraging attendance and facilitating their referral to pediatric dental services.

Medical and dental professionals who treat medically compromised children need to work together as a team, and adopt a multidisciplinary approach in order to optimally contribute to the child's quality of life.
A regular dental check-up should be included in treatment protocols to eliminate possible sources of infection. It is also very important to educate parents/patients about oral hygiene procedures.

\section{References}

[1] Brown A. Caries prevalence and treatment needs of healthy and medically compromised children at a tertiary care institution in Saudi Arabia. Eastern Mediterranean Health Journal, 2009, Vol. 15, No. 2: 378-86.

[2] Foster H, Fitzgerald J. Dental disease in children with chronic illness. Arch Dis Child 2005; 90(7): 703-708.

[3] Othman NAA, Sockalingam SNM and Mahyudd A. Oral health status in children and adolescents with haemophilia. Haemophilia 2015, 21(5): 605-611.

[4] Dhanuthai K, Sappayatosok K, Bijaphala P, Kulvitit S, Sereerat T. Prevalence of medically compromised conditions in dental patients. Med Oral Patol Oral Cir Bucal. 2009, Jun 1; 14 (6): E287-91.

[5] Mohammad SH, Syed KB, Al Harthi SMH, Al Qahtani KM, Abohasel SAS, Bagi AM. Prevalence of medical conditions among patients visiting dental school in Asir region, Saudi Arabia: a retrospective study. Gulf Medical Journal. 2016; 5(1): 21-26.

[6] Martins Esteves HJ, Suarez Quintanilla JM. Identification of medically compromised dental patients in Portuguese population. Oral Health Prev Dent 2013; 11(4): 315-322.

[7] Al-Bayaty HF, Murti PR, Naidu RS, Matthews R, Simeon D. Medical problems among dental patients at the school of dentistry, the university of the West Indies. J Dent Educ. 2009, Dec; 73(12): 1408-14.

[8] Radfar L, Suresh L. Medical profile of a dental school patient population. Journal of dental education 2007, 71(5): 682-686.

[9] Khader YS, Alsaeed O, Burgan SZ, Amarin ZO. Prevalence of Medical Conditions Among Patients Attending Dental Teaching Clinics in Northern Jordan. J Contemp Dent Pract 2007; 8(1): 60-67.

[10] Kaur J. Assessing the Medical Health Statistics of patients in a Dental Institute. J Adv Med Dent Scie Res 2017; 5(9): 64-68.

[11] Çetingüç A, Tekçiçek M, Güngör HC. Oral Health Status of Medically Compromised Children Referred for Dental Consultations: A Retrospective Study. Hacettepe Dişhek Fak Derg 2004, 28(3): 41-45.

[12] Schwarz JN, Monti A, Savelli-Castillo I, Nelson LP. Accuracy of Familial Reporting of a Child's Medical History in a Dental Clinic Setting. Pediat Dent, 2004; 26(5): 433-39.

[13] Abraham-Inpijn L, Russell G, Abraham DA and Al. A patient administered Medical Risk Related History questionnaire (EMRRH) for use in 10 European countries (multicenter trial). Oral Surg Oral Med Oral Pathol Oral Radiol Endod 2008; 105(5): 597-605.

[14] World Health Organization. Oral Health Surveys. Basic method. 4th ed. Geneva: World Health Organization; 1997 
[15] SILNESS J, LÖE H. Periodontal disease in pregnancy II. Correlation between oral hygiene and periodontal condition. Acta odontologica scandinavica, 1964, 22(1): 121-135.

[16] Joybell C, Krishnan R, Kumar S. Comparison of Two Brushing Methods-Fone's vs Modified Bass Method in Visually Impaired Children Using the Audio Tactile Performance (ATP) Technique. J Clin Diagn Res. JCDR 2015 Mar; 9(3): ZC19-ZC22.

[17] Parry JA, Khan FA. Provision of dental care for medically compromised children in the UK by general dental practitioners. Int j of paediatr dent, 2000, 10(4): 322-77.
[18] UFSBD. Nouvelles recommandations L'UFSBD réactualise ses stratégies de prévention. Pratique dentaire. Novembre 2013; 17-39. Available on March 10, 2019 at http://www.ufsbd.fr/wp-content/uploads/2013/11/Recosespace-PRO.pdf

[19] Hicks MJ, Flaitz CM. Epidemiology of dental caries in pediatric and adolescent population: a review of past and current trends. J Clinic Ped Dent 1993; 18(1): 43-49. 